\title{
Sodium glycididazole enhances the efficacy of combined iodine-125 seed implantation and chemotherapy in patients with non small-cell lung cancer
}

\author{
QING ZHANG ${ }^{*}$, DAO-QING WANG ${ }^{*}$ and YU-FEN WU
}

Department of Radiology, Affiliated Hospital of Shandong Academy of Medical Sciences, Jinan, Shandong 250031, P.R. China

Received June 2, 2014; Accepted February 13, 2015

DOI: $10.3892 / \mathrm{ol} .2015 .3039$

\begin{abstract}
The aim of the present study was to investigate the efficacy and safety of combined therapy with iodine-125 seed implantation and the gemcitabine plus cisplatin chemotherapeutic regimen, as well as treatment with the radiosensitizer sodium glycididazole (CMNa), in patients with non-small cell lung cancer (NSCLC). The 40 patients with NSCLC in the experimental group (19 females; mean age, $52.3 \pm 11.5$ years; age range, 34-74 years) received the combined therapy and CMNa, and the 41 controls (13 females; mean age, 53.7 \pm 10.7 years; age range, 8-79 years) received the combined therapy only. The response rate in the experimental group was significantly higher than in the control group (85.5 vs. $63.4 \% ; \mathrm{P}=0.027$ ), with no apparent complications. Therefore, it was concluded that such therapy may be reliable and well-tolerated for the treatment of patients with NSCLC.
\end{abstract}

\section{Introduction}

Lung cancer is one of the most common malignancies and the leading cause of tumor-associated mortality worldwide (1). Non-small cell lung cancer (NSCLC) is the most common form of lung cancer, however, its successful treatment remains difficult as $<20 \%$ of patients are candidates for curative surgical resection when NSCLC is initially diagnosed (2). For patients who cannot undergo surgery or who reject surgery, chemoradiotherapy is an alternative. The Department of Radiology at the Affiliated Hospital of Shandong Academy of Medical Sciences (Jinan, China) often employs combined therapy with computed tomography (CT)-guided radioactive iodine-125 ( $\left.{ }^{125} \mathrm{I}\right)$ seed implantation and a gemcitabine plus

Correspondence to: Professor Yu-Fen Wu, Department of Radiology, Affiliated Hospital of Shandong Academy of Medical Sciences, 38 Wuyingshan Road, Jinan, Shandong 250031, P.R. China E-mail: scimkr@163.com

*Contributed equally

Key words: carcinoma, non-small cell lung cancer, implant radiotherapy, sodium glycididazole, chemotherapy cisplatin (GP) chemotherapy regime for patients with advanced NSCLC. Compared with conventional external-beam radiotherapy, CT-guided ${ }^{125}$ I seed implantation can allow the application of an increased radiation dose without damaging organs adjacent to the target tumor; therefore, this technique is widely used for its curative effect, minimal surgical trauma and small number of complications $(3,4)$.

Chemotherapy with GP is a first-line treatment strategy for advanced NSCLC. A randomized study comparing the efficacy and safety of chemotherapeutic agents for advanced NSCLC indicated that treatment with GP was associated with a significantly longer time to the progression of disease, and higher 1- and 2-year survival rates (5).

Sodium glycididazole [CMNa $\left.\left(\mathrm{C}_{18} \mathrm{H}_{22} \mathrm{~N}_{7} \mathrm{NaO}_{10} \cdot 3 \mathrm{H}_{2} \mathrm{O}\right)\right]$, a newly synthesized radio- and chemotherapy sensitizer, is widely used in China. A number of previous studies have demonstrated positive results regarding the application of $\mathrm{CMNa}$ as a sensitizer for conventional external radiation or chemotherapy alone (6-10). Additionally, a number of studies have reported that concurrent chemoradiotherapy combined with CMNa may enhance the response rate and prolong the survival of patients with advanced esophageal, nasopharyngeal or laryngeal carcinoma $(8,10,11)$. However, to the best of our knowledge, the effect of CMNa on CT-guided ${ }^{125} \mathrm{I}$ seed implantation combined with chemotherapy has yet to be described in patients with NSCLC.

Therefore, the present study investigated the short-term efficacy and safety of combined therapy with CT-guided ${ }^{125}$ I seed implantation and GP regimen chemotherapy, in combination with CMNa administration, in patients with NSCLC.

\section{Patients and methods}

Study population. Between May and October 2013, 81 consecutive patients with NSCLC from the Affiliated Hospital of Shandong Academy of Medical Sciences (Jinan, China) were enrolled in the present study. The inclusion criteria were as follows: i) NSCLC confirmed by needle biopsy, fiberoptic bronchoscopy or cast-off cell examination; ii) stage III-IV NSCLC according to the International Union Against Cancer staging system (2009) (12); iii) ineligible for surgical resection or rejected surgical treatment for personal reasons; iv) newly diagnosed with cancer or had not received anti-tumor therapy within three months of undergoing the study treatment regime; 
Table I. Baseline characteristics of experimental and control group patients receiving therapy for non small-cell lung cancer.

\begin{tabular}{|c|c|c|c|}
\hline Characteristic & Experimental group $(n=40)$ & Control group $(n=41)$ & P-value \\
\hline Age, years ${ }^{a}$ & $52.3 \pm 11.5(34-74)$ & $53.7 \pm 10.7(38-79)$ & 0.576 \\
\hline Tumor size, $\mathrm{cm}^{\mathrm{a}}$ & $5.2 \pm 1.1(3.3-7.3)$ & $4.9 \pm 1.1(3.2-7.2)$ & 0.317 \\
\hline Gender, $\mathrm{n}$ & & & 0.146 \\
\hline Male & 21 & 28 & \\
\hline Female & 19 & 13 & \\
\hline Malignancy, n & & & 0.850 \\
\hline Squamous & 18 & 16 & \\
\hline Adenocarcinoma & 19 & 22 & \\
\hline Other & 3 & 3 & \\
\hline UICC TNM stage, $\mathrm{n}$ & & & 0.241 \\
\hline III & 30 & 35 & \\
\hline IV & 10 & 6 & \\
\hline
\end{tabular}

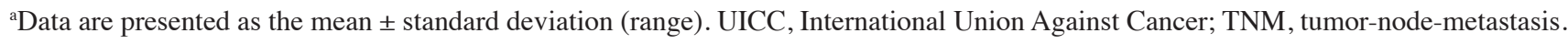

and v) liver and kidney function, as well as routine blood and coagulation function, within the normal ranges. Patients with uncontrolled infection, heart failure, respiratory failure or other concomitant serious disease contraindicating the procedure were excluded from the present study.

Patients were randomly divided into two groups for treatment: An experimental group and a control group. The 40 experimental patients (19 females; mean age, $52.3 \pm 11.5$ years; range, 34-74 years) received the combined therapy of CT-guided ${ }^{125}$ I seed implantation and GP regimen chemotherapy, as well as $\mathrm{CMNa}$ administration, and the 41 control patients (13 females; mean age, 53.7 \pm 10.7 years; range, 38-79 years) received the combined therapy only. The two groups did not significantly differ in any of the baseline characteristics investigated (Table I).

The present study was approved by the Institutional Ethics Committee of the Affiliated Hospital of Shandong Academy of Medical Sciences. Written informed consent was obtained from each patient following a full explanation of the nature of the current protocols.

${ }^{125}$ I seed implantation. Within 1 week prior to treatment, a baseline thoracic CT scan (SOMATOM Sensation 64; Siemens Healthcare, Erlangen, Germany) was performed on all patients. The CT images $(120 \mathrm{kV}, 250 \mathrm{~mA}, 300-\mathrm{mm}$ field of view and 5-mm slice thickness) were imported to the Fudan Brachytherapy Treatment Planning Computer System v1.0 (Fudan University, Shanghai, China) to create an individual treatment strategy for each patient. The gross tumor volume (GTV) was outlined and the planning treatment volume (PTV) included the entire GTV with $0.5-1.0-\mathrm{cm}$ margins. The primary planning goal was adequate PTV coverage with the prescribed radiation dose; therefore, a dose of 100-140 Gy was prescribed according to the location of each tumor. The system automatically calculated the expected number of ${ }^{125}$ I seeds (Shanghai Xinke Medical Company Co., Ltd., Shanghai, China) required and optimized their spatial distribution. The physician adjusted the number of seeds and their locations based on the isodose curves to achieve optimal dose conformity and safety. The ${ }^{125}$ I seeds (Xinke Medical Company Co., Ltd., Shanghai, China) were $4.5 \mathrm{~mm}$ long and $0.8 \mathrm{~mm}$ thick, with a half-life of 59.6 days, a penetration depth of $17 \mathrm{~mm}$, an incipient rate of $7 \mathrm{cGy} / \mathrm{h}$ and activity of $0.5-0.8 \mathrm{mCi}$.

All interventional procedures were performed in a standard CT room using a CT scanner. Following $\geq 6 \mathrm{~h}$ of fasting, the patient was placed in the supine, prone or lateral position, according to the position of the tumor, and was trained to ensure steady breathing during the procedure. The pathway through which the implantation needles reached the tumor was determined on the CT image, avoiding critical structures, such as vital blood vessels, major bronchi, the esophagus or the spinal cord. Subsequently, one or more 18-gauge needles were inserted percutaneously into the tumor with the patient under local anesthesia. A repeat CT scan facilitated the adjustment of the depth and angle of needle direction. Adjacent implantation needles were separated by $\sim 1 \mathrm{~cm}$. A turntable implantation gun (Xinke Medical Company Co., Ltd.) was loaded, and 2-5 ${ }^{125} \mathrm{I}$ seeds were released along the path at $\sim 1-\mathrm{cm}$ intervals when the needle was withdrawn. The distance between the ${ }^{125} \mathrm{I}$ seeds and the boundary of the lesion was maintained at $0.5-1.0 \mathrm{~cm}$, and the ${ }^{125} \mathrm{I}$ seeds remained $\geq 1 \mathrm{~cm}$ away from the aforementioned critical structures. The needles were removed only when all the seeds had been inserted in the correct position. The mean number of ${ }^{125} \mathrm{I}$ seeds implanted in each patient was $41.7 \pm 11.5$ (range, $20-74{ }^{125} \mathrm{I}$ seeds) and $42.2 \pm 8.0$ (range, 27-55) seeds for the experimental and control patients, respectively. CMNa and the first cycle of GP were administered 5-7 days after the interventional procedure in patients exhibiting no implantation-associated complications.

Chemotherapy. Two cycles of chemotherapy consisting of gemcitabine $\left(1,000 \mathrm{mg} / \mathrm{m}^{2}\right.$ on days 1 and 8$)$ and cisplatin $\left(30 \mathrm{mg} / \mathrm{m}^{2}\right.$ on days 1,2 and 3) were administered intravenously to each patient 5-7 days after ${ }^{125}$ I seed implantation. Each treatment cycle lasted 3 weeks. All patients received prophylactic agents consisting of the 5-HT3 antagonist 
metoclopramide, berbamine and dexamethasone. Supportive care, including the administration of antibiotics or analgesics, was provided if considered appropriate by the investigators. Adverse events were treated symptomatically and all chemotherapy was performed successfully.

CMNa administration. Between 5 and 7 days after ${ }^{125}$ I seed implantation, CMNa (LifeTech Pharmaceutical Ltd., Guangzhou, Guangdong, China) was administered to each patient in the experimental group. The method and dose were identical to that used with external beam radiotherapy, as follows: CMNa (800 mg/m² dissolved in $100 \mathrm{ml}$ physiological saline) administered intravenously within $30 \mathrm{~min}$, three times a week (on alternate days) for 8 weeks.

Follow-up and evaluation. All patients were followed up for 3-8 months (mean, 5.4 \pm 1.6 months). Within 1 week prior to treatment, all patients underwent a baseline thoracic CT. Follow-up CT examinations were performed monthly in the first 3 months and subsequently at 2 -month intervals. The short-term curative effects were graded according to the Response Evaluation Criteria in Solid Tumors (13) as follows: Complete response (CR) was defined as the disappearance of all target lesions; partial response (PR) was defined as a $\geq 30 \%$ decrease in the sum of the longest diameters of the target lesions; stable disease (SD) was defined as a $<30 \%$ reduction or $<20 \%$ increase in the sum of the longest diameters of the target lesions; and progressive disease (PD) was defined as a $\geq 20 \%$ increase in tumor load or the development of new lesions in a previously uninvolved site. Furthermore, response rate was defined as the sum of CR and PR. Following implantation, liver and kidney function tests, routine blood tests and electrocardiography (ECG) were performed every 2 weeks in the first 3 months, or as indicated. Complications associated with the implantation procedure and brachytherapy were investigated. In addition, adverse events (grades 1 to 5) associated with chemotherapy or CMNa were evaluated according to the Common Terminology Criteria for Adverse Events (version 4.0) (14) of the US National Cancer Institute.

Statistical analysis. All numerical data were expressed as the mean \pm standard deviation and determined by performing independent-samples t-tests between the two groups. Categorical variables at baseline and clinical efficacy were compared using the $\chi^{2}$ test, and adverse effects were evaluated by performing a $\chi^{2}$ or Wilcoxon rank test. All significance tests were two-tailed, and $\mathrm{P}<0.05$ were considered to indicate a statistically significant difference. Data processing and analysis involved the use of SPSS software (version 15.0; SPSS Inc., Chicago, IL, USA).

\section{Results}

CR, PR, SD and PD were observed in 8, 26, 5 and 1 experimental patients, respectively, and 6, 20,9 and 6 control patients, respectively (Fig. 1). The response rates (sum of CR and PR) were 85.5 and $63.4 \%$ for the experimental and control groups, respectively $(\mathrm{P}=0.027)$.

A pneumothorax developed in 17 experimental patients $(42.5 \%)$ and 14 control patients $(34.1 \%)$ during the procedures; therefore, $6(15.0 \%)$ and 7 (17.1\%) patients,

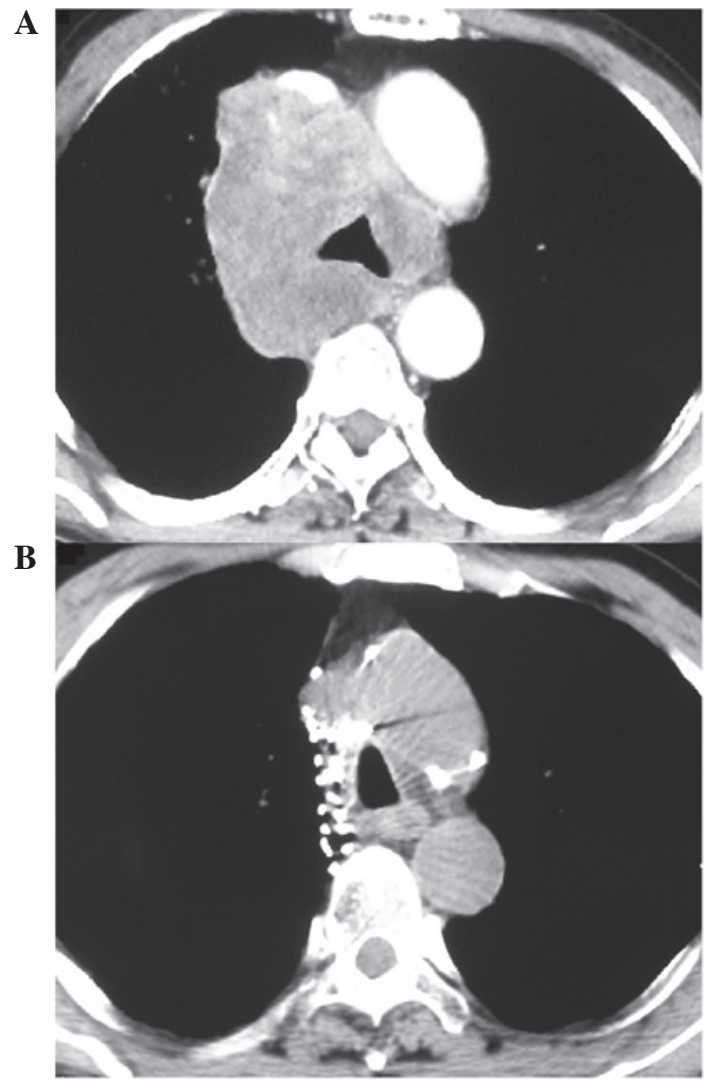

Figure 1. Computed tomography (CT) scan of a 64-year-old male patient with non-small cell lung cancer. The patient received combined therapy with iodine-125 seed implantation and a gemcitabine plus cisplatin chemotherapyregimen, as well as administration of the radiosensitizer sodium glycididazole. (A) Baseline CT image prior to treatment; and (B) CT scan performed five months after treatment, indicating a complete response.

respectively, underwent puncture aspiration or closed thoracic drainage for 1-2 days to relieve the complication. The remaining patients exhibited no apparent symptoms and therefore received no treatment, as the pneumothorax was mild. At 3 days post-treatment, all patients demonstrated complete regression of the pneumothorax. Furthermore, 5 (12.5\%) and 9 (22.0\%) experimental and control patients, respectively, demonstrated hemoptysis or focal hemorrhage, as detected by CT. The two groups did not significantly differ in the rate of pneumothorax and hemoptysis or focal hemorrhage $(\mathrm{P}=0.439$ and $\mathrm{P}=0.261$, respectively). No lethal hemoptysis or hemorrhage occurred, and no ${ }^{125}$ I seeds migrated to other tissues or organs. Additionally, no patients appeared to exhibit radiation pneumonia or radiation esophagitis.

Thus, the two groups did not significantly differ in the occurrence of adverse events associated with chemotherapy or CMNa (Table II). Adverse events of grade 3 were treated using routine clinical methodology. No adverse events of grade 4 or above were identified; therefore, chemotherapy was not delayed or canceled in any of the cases.

\section{Discussion}

The present study evaluated the short-term efficacy and safety of combined therapy with CT-guided radioactive ${ }^{125} \mathrm{I}$ seed implantation and GP regimen chemotherapy, as well as $\mathrm{CMNa}$ 
Table II. Adverse events observed in the experimental and control groups upon follow-up.

\begin{tabular}{|c|c|c|c|c|c|c|c|c|c|c|c|c|c|}
\hline \multirow[b]{3}{*}{ Adverse event } & \multicolumn{12}{|c|}{ Grade } & \multirow[b]{3}{*}{ P-value } \\
\hline & \multicolumn{6}{|c|}{ Experimental group $(\mathrm{n}=40)$} & \multicolumn{6}{|c|}{ Control group $(n=41)$} & \\
\hline & 0 & 1 & 2 & 3 & 4 & 5 & 0 & 1 & 2 & 3 & 4 & 5 & \\
\hline White blood cell decrease & 20 & 10 & 8 & 2 & 0 & 0 & 24 & 8 & 7 & 2 & 0 & 0 & 0.505 \\
\hline Platelet count decrease & 19 & 9 & 9 & 3 & 0 & 0 & 20 & 11 & 8 & 2 & 0 & 0 & 0.726 \\
\hline Alanine aminotransferase increase & 17 & 14 & 5 & 4 & 0 & 0 & 20 & 13 & 6 & 2 & 0 & 0 & 0.536 \\
\hline Creatinine increase & 21 & 9 & 8 & 2 & 0 & 0 & 25 & 10 & 4 & 2 & 0 & 0 & 0.350 \\
\hline Vomiting & 13 & 17 & 8 & 2 & 0 & 0 & 15 & 15 & 10 & 1 & 0 & 0 & 0.833 \\
\hline Palpitations & 32 & 5 & 3 & 0 & 0 & 0 & 36 & 4 & 1 & 0 & 0 & 0 & 0.318 \\
\hline Sinus tachycardia & 31 & 6 & 2 & 1 & 0 & 0 & 35 & 3 & 3 & 0 & 0 & 0 & 0.390 \\
\hline
\end{tabular}

administration, in patients with advanced NSCLC. This treatment strategy produced greater efficacy compared with the control treatment of combined therapy alone, and the two groups did not significantly differ in the rate of adverse events.

Interstitial permanent brachytherapy is based on the implantation of radioactive sources inside or close to the tumor target, thereby delivering high-dose radiation directly to the tumor, while sparing the surrounding healthy tissues, as a sharp dose drop-off occurs outside of the implanted target area. Thus, percutaneous CT-guided ${ }^{125}$ I seed implantation has become a treatment focus due to its ability to increase the possible radiation dose to tumors without damage to neighboring organs (15). Using this method, highly effective radiation doses can be applied as a single fraction. Compared with fractionated external radiotherapy, which is effective only to tumor cells in specific phases of the cell cycle, the ${ }^{125}$ I seed continuously generates a low level of radiation sufficient to kill the tumor cells or cause them to lose the ability to reproduce, thus providing protracted cell killing for up to several weeks or months (16). Additionally, by providing continuous low-dose radiation, ${ }^{125}$ I seeds may decrease the oxygen enhancement ratio, thus improving efficacy in hypoxic regions of the tumor (17). Previous studies investigating this strategy for the treatment of liver cancer and other malignancies demonstrated that ${ }^{125} \mathrm{I}$ brachytherapy is safe and may provide long-term local control and increased survival $(4,18)$.

GP is a standard chemotherapeutic regimen for the first-line treatment of advanced NSCLC. Chemotherapy with GP may result in a higher overall survival rate for patients with advanced NSCLC when compared with other cisplatin-based regimens (19). A randomized study comparing the efficacy and safety of various chemotherapy agents for advanced NSCLC indicated that treatment with GP was associated with a significantly longer time to the progression of disease, and higher 1- and 2-year survival rates (5).

The treatment of advanced NSCLC is based on local and systemic control of the disease (20). CT-guided ${ }^{125}$ I seed implantation may improve the local control rate (21), and chemotherapy potentially exhibits an effect on distant metastasis (22). Therefore, the combination of brachytherapy and chemotherapy is considered to be superior to either alone.

$\mathrm{CMNa}$, a novel nitroimidazole compound, is the firsthypoxic sensitizer synthesized in China and is widely used (6-9). The mechanism of CMNa involves the selective potentiation of DNA duplex damage caused by chemoradiotherapy in hypoxic cells, and the inhibition of DNA polymerase B activity to inhibit the recovery of potentially lethal and sub-lethal cellular damage. Previous studies have demonstrated that CMNa exhibits an overt radiation-enhancing effect in vitro and in vivo $(6-9,23,24)$. In addition, a number of studies have indicated that CMNa may enhance the chemosensitivity of specific chemotherapeutic agents $(25,26)$. In the present study, the efficacy of ${ }^{125}$ I seed implantation combined with GP regimen chemotherapy and CMNa administration was significantly improved in the experimental group compared with the control group. Thus, CMNa may enhance the therapeutic effect of ${ }^{125}$ I seed implantation combined with GP regimen chemotherapy for the treatment of patients with NSCLC.

The two groups experienced no serious complications. In previous studies $(27,28)$, pneumothorax and hemoptysis were the predominant complications of ${ }^{125}$ I seed implantation. However, in the present study, the two groups did not significantly differ in the rates of pneumothorax and hemoptysis or focal hemorrhage, and all patients were successfully treated using appropriate therapies. Furthermore, no lethal hemoptysis occurred and no ${ }^{125}$ I seeds migrated to other tissues or organs. Due to a sharp dose drop-off outside of the implanted target area, ${ }^{125} \mathrm{I}$ seed implantation delivers a low dose of radiation to the healthy tissues surrounding the tumor; therefore, it does not typically result in serious complications (29). No patients in the current study appeared to exhibit radiation pneumonia, radiation esophagitis or other radiation-association complications upon follow-up. Thus, the safety of GP chemotherapy alone or combined with CMNa was considered to be acceptable. As in previous studies, the most common adverse reactions associated with $\mathrm{CMNa}$ in the present study were mild gastrointestinal duct reactions, including nausea, vomiting and constipation; mild reversible increases in alanine aminotransferase and bilirubin expression levels; and varying degrees of change in cardiac function and ECG results upon treatment with large doses of CMNa $(7,30,31)$. In the current study, the use of CMNa did not significantly increase the occurrence of adverse events associated with chemotherapy in the experimental group compared with the control group. 
Various factors affected the results of the present study. First, the heterogeneity of radiation distribution. Despite the assistance of the TPS, the positioning of all seeds in accurately remains difficult due to the complexity of the implantation procedure. Furthermore, the prescribed dose (seed number) varied depending on the location of the tumor. For example, in patients with NSCLC close to critical structures, such as vital blood vessels, the major bronchi, the esophagus and the spinal cord, a low radiation dose was prescribed. The ${ }^{125}$ I seeds are positioned $\geq 1 \mathrm{~cm}$ away from these critical structures due to the concern of possible complications, such as hemorrhage and bronchial injury. Therefore, regions of insufficient dose or cold spots remain in specific lesions. The heterogeneity of radiation distribution and the variety of prescribed doses affected the response of the tumor or in specific cases resulted in local relapse.

The second factor affecting the results of the present study was the method used to administer CMNa. CMNa was administered according to the clinical practice used with external beam radiotherapy. However, as opposed to conventional fractionated radiotherapy, ${ }^{125} \mathrm{I}$ seed implantation continuously generates a low level of radiation and provides protracted cell killing over a relatively long period of time. Thus, the optimal dose and method of administering CMNa requires additional investigation. The third factor was the interaction between $\mathrm{CMNa}$ and chemotherapeutic agents. These interactions also require further investigation, in particular, whether CMNa increases the toxicity of specific chemotherapeutic agents. The final affecting factors were the small sample size and the short follow-up time.

In conclusion, despite the aforementioned limitations, the preliminary results of the current study indicate that $\mathrm{CMNa}$ may enhance the short-term efficacy of combined therapy with ${ }^{125}$ I seed implantation and GP regimen chemotherapy for NSCLC. This strategy appears to be safe and effective for the treatment of patients with NSCLC. However, additional studies with larger samples and a longer follow-up period are required to validate the findings of the present study.

\section{Acknowledgements}

The authors would like to thank Dr. Yue Ren of the Department of Radiology at the Affiliated Hospital of Shandong Academy of Medical Sciences for providing assistance in performing the present study.

\section{References}

1. Devesa SS, Bray F, Vizcaino AP and Parkin DM: International lung cancer trends by histologic type: male:female differences diminishing and adenocarcinoma rates rising. Int J Cancer 117 294-299, 2005

2. Cardona AF, Reveiz L, Ospina EG, et al: Palliative endobronchial brachytherapy for non-small cell lung cancer. Cochrane Database Syst Rev: CD004284, 2008.

3. Ebara S, Katayama N, Tanimoto R, et al: Iodine-125 seed implantation (permanent brachytherapy) for clinically localized prostate cancer. Acta Med Okayama 62: 9-13, 2008.

4. Nag S, DeHaan M, Scruggs G, Mayr N and Martin EW: Long-term follow-up of patients of intrahepatic malignancies treated with iodine-125 brachytherapy. Int J Radiat Oncol Biol Phys 64: 736-744, 2006.

5. Schiller JH, Harrington D, Belani CP, et al: Comparison of four chemotherapy regimens for advanced non-small-cell lung cancer. N Engl J Med 346: 92-98, 2002.
6. Shen G and Bao Y; Sodium Glycididazole Clinical Trial Cooperative Group: Double blind randomized clinical trail phase II on the sensitization by sodium glycididazole injection. Chin J Radiat Oncol 12: 254-258, 2003 (In Chinese)

7. Wang XP, Yun S, Zhang XL and Ding W: Primary clinical study of radiosensitizer CMNa. Chin Clin Oncol 8: 28-32, 2003 (In Chinese).

8. Zeng YC, Wu R, Xu ZG, et al: Safety and radiation-enhancing effect of sodium glycididazole in locoregionally advanced laryngeal cancers previously treated with platinum-containing chemotherapy regimens: A preliminary report. Cancer Radiother 14: 59-64, 2010.

9. Liu MZ, He LR, Lu TX, et al: Effect of hypoxic radiosensitizer sodium glycididazole on long-term result of radiotherapy for nasopharyngeal carcinoma. Chin J Oncol 28: 932-937, 2006 (In Chinese).

10. Yang J, Liu MZ, Cai L, et al: Phase II clinical trial of sodium glyci-didazole (CM-Na) combined with concurrent radiochemotherapy for advanced esophageal carcinoma. Chin J Cancer: 622-626, 2008 (In Chinese).

11. He ZY, Li FY, Tong Q, Liao ZW, Guan XX and Wang W: Concurrent chemoradiotherapy with sodium glycididazole and cisplatin for local advanced nasopharyngeal carcinoma. J First Mil Med Univ 28: 2038-2040, 2008 (In Chinese).

12. International Union Against Cancer (UICC): Lung and Pleural Tumours. In: TNM Classification of Malignant Tumours. Sobin LH, Gospodarowicz MK and Wittekind C (eds). 7th edition. Wiley-Blackwell, New York, NY, pp99-106, 2009.

13. Eisenhauer EA, Therasse P, Bogaerts J, et al: New response evaluation criteria in solid tumours: revised RECIST guideline (version 1.1). Eur J Cancer 45: 228-247, 2009.

14. National Cancer Institute: Common Terminology Criteria for Adverse Events (CTCAE) version 4.0. Accessed June 14, 2010.

15. Hu XK, Wang MY, Yang ZG, et al: The study of curing central-type lung cancer by $\mathrm{CT}$ guided percutaneous embedding of ${ }^{125}$ I seeds. Chin J Radiol 38: 910-915, 2004 (In Chinese).

16. Ling CC: Permanent implants using Au-198, Pd-103 and I-125: radiobiological considerations based on the linear quadratic model. Int J Radiat Oncol Biol Phys 23: 81-87, 1992.

17. Blasko JC, Mate T, Sylvester JE, Grimm PD and Cavanagh W: Brachytherapy for carcinoma of the prostate: techniques, patient selection and clinical outcomes. Semin Radiat Oncol 12: 81-94, 2002.

18. Henry AM, Al-Qaisieh B, Gould K, et al: Outcomes following iodine-125 monotherapy for localized prostate cancer: the results of Leeds 10-year single-center brachytherapy experience. Int J Radiat Oncol Biol Phys 76: 50-56, 2010.

19. Kubota K, Nishiwaki Y, Ohashi Y, et al: The Four-Arm Cooperative Study (FACS) for advanced non-small cell lung cancer (NSCLC). J Clin Oncol (Meeting Abstracts) 22 (Suppl): 7006, 2004

20. Vokes EE, Choy H, Gandara D and Mattson K: Adjuvant and neoadjuvant treatments for NSCLC. Lung Cancer 38 (Suppl 4): 29-35, 2002.

21. Chen A, Galloway M, Landreneau R, et al: Intraoperative 125I brachytherapy for high-risk stage I non-small cell lung carcinoma. Int J Radiat Oncol Biol Phys 44: 1057-1063, 1999.

22. Depierre A, Milleron B, Moro-Sibilot D, et al; French Thoracic Cooperative Group: Preoperative chemotherapy followed by surgery compared with primary surgery in resectable stage I (except T1N0), II, and IIIa non-small-cell lung cancer. J Clin Oncol 20: 247-253, 2002.

23. Zheng X, Meng X, Zhao F, LI Y and GAO J: The in situ tumor response to radiosensitization of a novel sensitizer sodium glycididazole. J Radiat Res Radiat Process 18: 91-95, 2000 (In Chinese).

24. Zhang X, Ji HB, Chen ZH, OuYang XN and Yang RJ: Lung cancer radiosensitization by $\mathrm{CMNa}$ in vitro and in vivo. Chin $\mathrm{J}$ Radiol Med and Prot 25: 316-318, 2005 (In Chinese).

25. Lei KJ, Wang J, Jia YM and Cao Y Y: Chemo-sensitivity-enhancing effects of low dose glycididazolum natrium on patients with advanced non-small cell lung cancer during intervention. Chinese-German J Clin Oncol 9: 385-387, 2010 (In Chinese).

26. Lei KJ, Jia YM, Wang J and Du YP: Effects of CMNa combined with paclitaxel and carboplatin on elevated CA125 levels in post-operation advanced ovarian cancer patients. Chinese-German J Clin Oncol 7: 600-602, 2008 (In Chinese).

27. Brach B, Buhler C, Hayman MH and Joyner LR Jr and Liprie SF: Percutaneous computed tomography-guided fine needle brachytherapy of pulmonary malignancies. Chest 106: 268-274, 1994. 
28. Martínez-Monge R, Pagola M, Vivas I and López-Picazo JM: CT-guided permanent brachytherapy for patients with medically inoperable early-stage non-small cell lung cancer (NSCLC). Lung Cancer 61: 209-213, 2008.

29. Lewis JW Jr, Ajlouni M, Kvale PA, et al: Role of brachytherapy in the management of pulmonary and mediastinal malignancies. Ann Thorac Surg 49: 728-733, 1990.
30. Fu LQ, Huang F, Guo JH, et al: Phase I clinical pharmacokinetics of glycididazolum natrium. Chin J Pharm Toxicol 18: 87-92, 2004 (In Chinese).

31. Gao HJ, Shen G, Wang HM, et al: Phase I clinical trail of sodium glycididadole (CMNa) in cancer patients. Chin J New Drugs 13: 260-263, 2004 (In Chinese). 